Article

\title{
Features of the High-Temperature Structural Evolution of GeTe Thermoelectric Probed by Neutron and Synchrotron Powder Diffraction
}

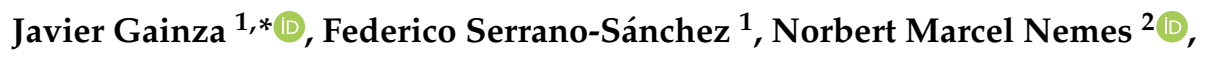 \\ José Luis Martínez ${ }^{1}$ (D), María Teresa Fernández-Díaz ${ }^{3}$ and José Antonio Alonso ${ }^{1, *(D)}$ \\ 1 Instituto de Ciencia de Materiales de Madrid (ICMM), Consejo Superior de Investigaciones \\ Científicas (CSIC), Sor Juana Inés de la Cruz 3, 28049 Madrid, Spain; fserrano@icmm.csic.es (F.S.-S.); \\ martinez@icmm.csic.es (J.L.M.) \\ 2 Departamento de Física de Materiales, Universidad Complutense de Madrid, E-28040 Madrid, Spain; \\ nmnemes@fis.ucm.es \\ 3 Institut Laue Langevin, BP 156X, Grenoble F-38042, France; ferndiaz@ill.fr \\ * Correspondence: j.gainza@csic.es (J.G.); ja.alonso@icmm.csic.es (J.A.A.)
}

Received: 26 November 2019; Accepted: 24 December 2019; Published: 25 December 2019

\begin{abstract}
Among other chalcogenide thermoelectric materials, GeTe and derivative alloys are good candidates for intermediate temperature applications, as a replacement for toxic $\mathrm{PbTe}$. We have prepared pure polycrystalline GeTe by using arc-melting, and investigated its structural evolution by using neutron powder diffraction (NPD) and synchrotron X-ray diffraction (SXRD), as well as its correlation with the thermal variation of the Seebeck coefficient. Besides a significant Ge deficiency ( $7 \%$ Ge vacancies), the thermal evolution of the unit-cell volume and Ge-Te bond lengths in the rhombohedral phase (space group $R 3 \mathrm{~m}$ ), below $700 \mathrm{~K}$, show unexpected anomalies involving the abrupt $\mathrm{Ge}-\mathrm{Te}$ bond lengthening accompanied by increased Te thermal displacements. Above $700 \mathrm{~K}$, the sample is cubic (space group Fm-3m) and shows considerably larger displacement parameters for $\mathrm{Ge}$ than for $\mathrm{Te}$, as a consequence of the random distribution of the lone pair lobes of $\mathrm{Ge}^{2+}$. The Seebeck coefficient, reaching $120 \mu \mathrm{V} \mathrm{K}^{-1}$ at $775 \mathrm{~K}$, shows a shoulder in the $500-570 \mathrm{~K}$ region that can be correlated to the structural anomaly, modifying the electron-phonon scattering in this temperature range.
\end{abstract}

Keywords: thermoelectrics; neutron powder diffraction; Ge deficiency; structural phase transition

\section{Introduction}

Group IV-VI materials and their alloys display an extensive set of functionalities of scientific and technological interest [1,2]. Among them, Germanium Telluride (GeTe) derivatives have been employed in phase-change non-volatile memory optoelectronic devices and considered for thermoelectric applications [3-5]. The implementation of GeTe materials in thermoelectric devices has been investigated as a possible substitute for $\mathrm{PbTe}$. Owing to their excellent electrical transport properties, the thermal transport of GeTe materials have been optimized to show a high-thermoelectric figure of merit, evaluated as $z T=\frac{S \sigma}{\kappa} T$, where $T$ is the absolute temperature, $S$ is the Seebeck coefficient, $\sigma$ is the electrical conductivity, and $\kappa$ is the total thermal conductivity [6-9]. The parent GeTe compound presents high-carrier mobilities and concentration $[10,11]$, which are tuned by dopants such as $\mathrm{In}, \mathrm{Bi}$, and $\mathrm{Sb}$, to reduce the carrier concentration to an optimum value in order to obtain high power factors and to reduce the lattice thermal conductivity by enhancing phonon scattering mechanisms [12-14]. This phonon scattering in GeTe is also promoted by domain boundaries due to the herringbone microstructure commonly found in the rhombohedral phase $[15,16]$. However, the implementation 
of GeTe in thermoelectric devices is limited by a structural phase transition due to the concomitant abrupt change in the thermal expansion and transport properties. The underlying mechanisms of this transition have been the aim of many investigations concerning the optimization for commercial devices, but still there are many features to clarify $[2,17]$.

At room temperature, pristine $\alpha$-GeTe presents a rhombohedral $R 3 m$ (No. 160) structure, which is described as a distortion of the rocksalt $\mathrm{NaCl}(\mathrm{PbTe})$ structure with pseudocubic $\alpha$ angle $\approx 88.3^{\circ}$. It is a narrow band gap ferroelectric with a degenerate p-type behavior due to the numerous Ge vacancies in the crystalline structure inducing positive electronic carriers. There is a particular distortion of Ge-Te bonds along the [111] direction yielding a net polarization component. This has been denoted as a Peierls distortion in the one dimensional line along the distorted p-bonds [17]. GeTe undergoes a structural phase transition to a cubic Fm-3m structure along with a ferroelectric to paraelectric transition at Tc $\sim 705 \mathrm{~K}$ [18]. This was described as a displacive second-order transition, as the $\mathrm{x}$ parameter deviation and $\alpha$ angle (in the rhombohedral setting) show a continuous variation for which the different lengths of the Ge-Te bonds gradually become equal. An inelastic neutron scattering study proposed this transition as a result of the condensation of three softened phonon modes, and further supported the displacive transition model [19]. Differences in the actual temperature and local mechanisms of the transition have been reported in several works, showing a dependence on the structural defects and nanodomains of the GeTe samples [19-21], and the order-disorder nature for the transition has been also proposed to be opposite to the displacive model [20,22]. The formation of Ge vacancies was reported as a crucial component in the transformation to the cubic phase and the linked anomalous sudden volume contraction [23]. Overall, the characteristic transport properties of GeTe have been ascribed to the Ge vacancies, resonant bonding [24], and structural distortion of the crystalline structure [25].

This work reports on an exhaustive structural characterization across the phase transition of GeTe using temperature-dependent high-resolution neutron synchrotron diffraction data. We observed an unreported anomaly in the thermal evolution of the rhombohedral phase concerning the unit-cell volume, bonding distances, and displacement parameters, which can be correlated to the thermoelectric power in the temperature range $500-570 \mathrm{~K}$.

\section{Experimental Section}

GeTe alloys were obtained as compact pellets directly from an Edmund Buhler mini-arc furnace. Mixtures of nominal 1:1 stoichiometric amounts of analytical grade Ge (Cerac, 99.999\%) and Te (Alpha, 99.99\%) were ground, pelletized, and molten under Ar atmosphere in a water-cooled Cu crucible, leading to intermetallic ingots. The as-grown ingots were sintered by spark plasma sintering (SPS) at $723 \mathrm{~K}$ for $5 \mathrm{~min}$ under an axial pressure of 40MPa (Dr. Sinter SPS-1050-CE, Fuji Electronic Industrial, Saitama, Japan) for transport characterization, or ground into powder for structural analysis. A laboratory XRD pattern was collected on a Bruker D5 diffractometer (Bruker, Karlsruhe, Germany) with $\mathrm{K}_{\alpha} \mathrm{Cu}(\lambda=1.5418 \AA)$ radiation. To study the crystallographic structure evolution, NPD patterns were collected at room temperature in the D2B instrument (Institute Laue Langevin, Grenoble, France) with a wavelength of $1.594 \AA$, at room-temperature $(298 \mathrm{~K}, \mathrm{RT})$ and at 523, 573, 633 and $683 \mathrm{~K}$. The powder sample (about $2 \mathrm{~g}$ ), contained in a V cylinder, was introduced in a vanadium furnace working under vacuum $\left(10^{-5} \mathrm{mbar}\right)$. The measurements lasted $2 \mathrm{~h}$ in the high-flux mode. In order to complement the high-temperature structural evolution, synchrotron X-ray powder diffraction (SXRD) patterns were collected at 303, 663, 703, 743, and $783 \mathrm{~K}$, in the MSPD diffractometer at the ALBA facility, Barcelona (Spain), selecting an incident beam with $28 \mathrm{keV}$ energy, $\lambda=0.4427 \AA$. The high angular resolution mode (MAD set-up) was selected [26]. The polycrystalline powder was contained in quartz capillaries of $0.7 \mathrm{~mm}$ diameter, which were rotating during the acquisition time. In both cases, the refinement of the structure was performed by the Rietveld method using the Fullprof software (version Sept 2018, Grenoble, France) [27,28]. A pseudo-Voigt function was chosen to generate the line shape of the diffraction peaks. The background was interpolated between regions devoid of 
reflections. The following parameters were refined in the final run: scale factor, background coefficients, zero-point error, pseudo-Voigt corrected for asymmetry parameters, positional coordinates, anisotropic displacement factors, and occupancy factors. For the neutron refinements, the coherent scattering lengths for Ge and Te were 8.185 and $5.80 \mathrm{fm}$, respectively. Differential scanning calorimetry (DSC) measurements were performed in a Mettler TA3000 system (Mettler-Toledo, Columbus, OH, USA) equipped with a DSC30 unit, in the temperature range from 50 to $540{ }^{\circ} \mathrm{C}$. The heating and cooling rates were $10{ }^{\circ} \mathrm{C} \mathrm{min}{ }^{-1}$, using about $70 \mathrm{mg}$ of sample. The Seebeck coefficient was measured in a home-made apparatus using niobium and chromel thermocouples [29].

\section{Results and Discussion}

Polycrystalline GeTe was obtained by carefully grinding the as-grown ingot from the arc furnace. The X-ray diffraction pattern collected with $\mathrm{Cu} \mathrm{K} \alpha$ radiation (Figure 1a) corresponds to the well-known rhombohedral structure, as identified via Rietveld refinement. No impurities or unreacted products were detected.
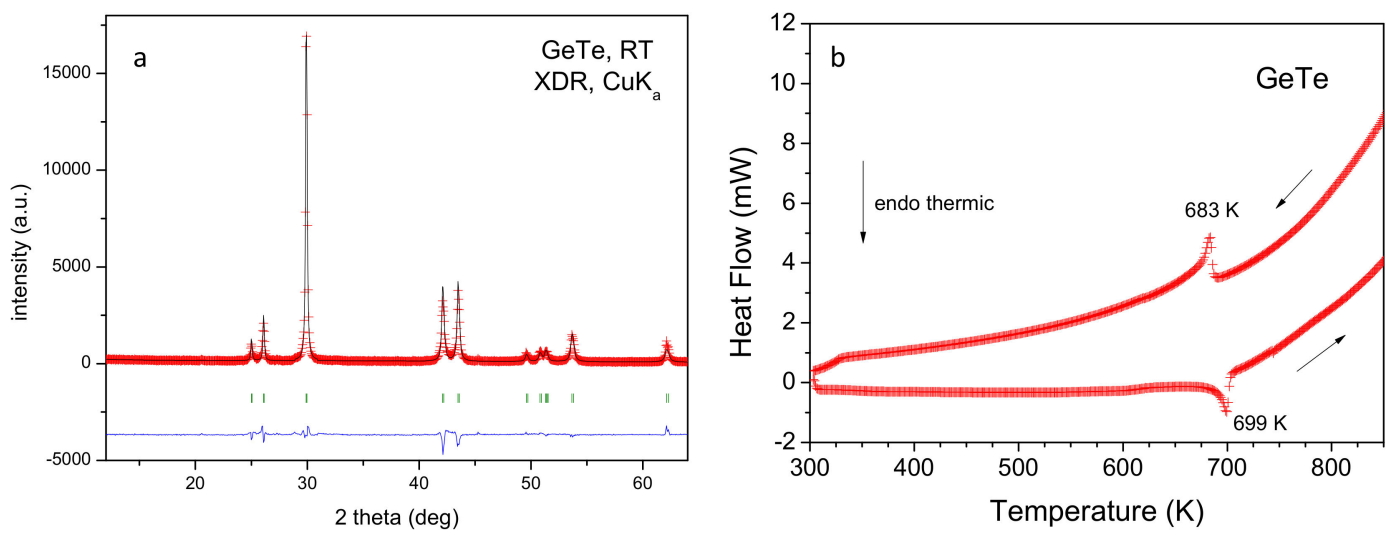

Figure 1. (a) XRD pattern of GeTe from laboratory XRD, displaying the characteristic splitting of the rhombohedral phase at RT; (b) Differential scanning calorimetry (DSC) curves, showing an endothermic peak in the heating run, and an exothermic peak in the cooling run.

The well-known structural transition from rhombohedral to cubic above room-temperature was previously characterized by DSC measurements. Figure $1 \mathrm{~b}$ displays the DSC curves for the heating and cooling runs. An endothermic peak at $699 \mathrm{~K}$ was observed during the heating run corresponding to the structural phase transition, while the cooling run presents an exothermic peak at $683 \mathrm{~K}$, showing a significant hysteretic effect suggesting a first-order transition. These results are in agreement with the previous thermal analysis data of GeTe [30], reporting $T_{t}=700 \mathrm{~K}$.

A neutron powder diffraction (NPD) study was essential for investigating the structural evolution when approaching the phase transition. NPD data were collected at 298, 523, 573, 633, and $683 \mathrm{~K}$, just below the structural transition, in order to unveil details of the structural arrangement in correlation with certain anomalies in the Seebeck coefficient thermal evolution, as described below. Neutrons take advantage of the access to a large region of the reciprocal space with no form factors, enabling them to decouple the displacement factors and occupancy factors during the structural refinement. The rhombohedral crystal structure was defined in the acentric $R 3 m$ space group (No. 160), $Z=3$, in the hexagonal setting, with unit-cell parameters at $298 \mathrm{~K}$ of a $=4.15871$ (9) $\AA$ and $\mathrm{c}=10.6731$ (3) $\AA$, and $\mathrm{V}=159.86(1) \AA^{3}$. The starting model was taken from Chattopadhyay et al. [18] Both Ge and Te atoms were located at $3 a(0,0, z)$ positions. The $z$ parameter for Ge was fixed to that given in reference [16], since the simultaneous refinement of $z$ for Ge and Te is not possible in an acentric space group. Anisotropic displacement factors were refined for both atoms; the occupancy factors of Ge (with respect to Te fixed to unity) gave a substantial departure from the full stoichiometry, as $\mathrm{Ge}_{0.927(15)} \mathrm{Te}$. Table 1 condenses the most important parameters after the Rietveld refinement. The same rhombohedral model was utilized to refine the crystal structures between $523 \mathrm{~K}$ and $683 \mathrm{~K}$. The rhombohedral distortion becomes 
weaker as it approaches the phase transition, which is manifested as a progressive disappearance of the characteristic splitting of some reflections. This is illustrated in the Rietveld plots included in Figure 2a at $523 \mathrm{~K}$, and Figure $2 \mathrm{~b}$ at $683 \mathrm{~K}$.
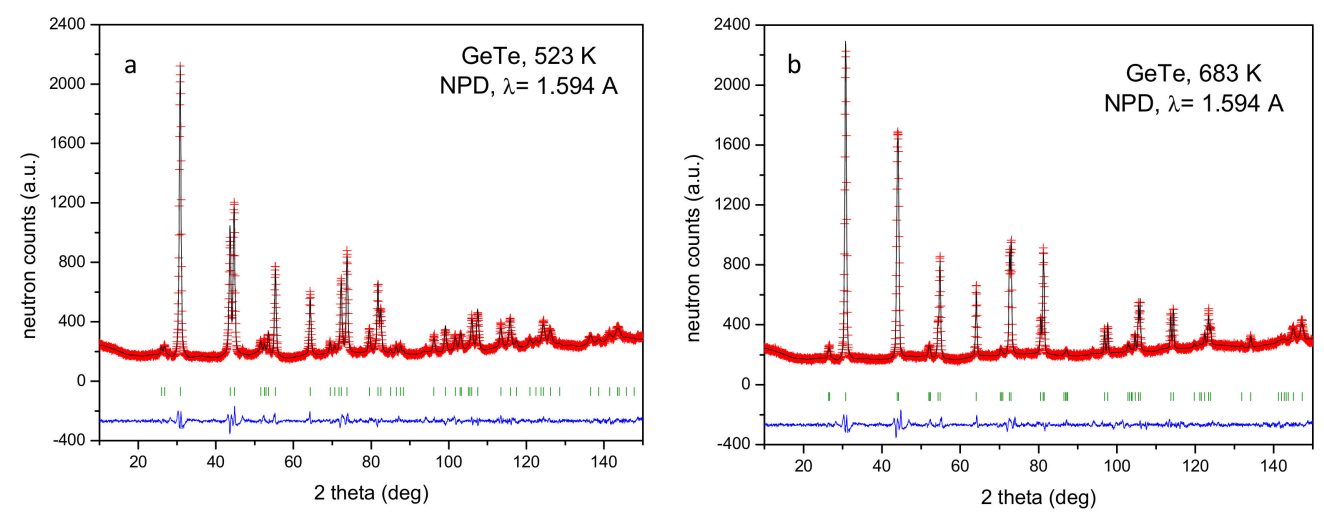

Figure 2. Neutron powder diffraction (NPD) profiles after the Rietveld refinement of the rhombohedral crystal structure at (a) $523 \mathrm{~K}$ and (b) $683 \mathrm{~K}$.

Table 1. Main structural parameters after the Rietveld refinement from NPD data for GeTe in the rhombohedral $(R 3 m)$ phase (hexagonal setting).

\begin{tabular}{cccccc}
\hline Temperature (K) & $\mathbf{2 9 8}$ & $\mathbf{5 2 3}$ & $\mathbf{5 7 3}$ & $\mathbf{6 3 3}$ & $\mathbf{6 8 3}$ \\
\hline$a / \AA$ & $4.15871(9)$ & $4.18835(16)$ & $4.20193(15)$ & $4.21864(12)$ & $4.23462(10)$ \\
$c / \AA$ & $10.6731(3)$ & $10.6155(6)$ & $10.5841(5)$ & $10.5258(4)$ & $10.4734(3)$ \\
$\mathrm{V} / \AA^{3}$ & $159.859(7)$ & $161.271(12)$ & $161.839(11)$ & $162.231(9)$ & $162.647(8)$ \\
$\mathrm{z}(\mathrm{Ge}) / \AA$ & 0.23760 & 0.23760 & 0.23760 & 0.23760 & 0.23760 \\
$\mathrm{f}_{\mathrm{occ}} \mathrm{Ge}$ & $0.927(15)$ & 0.927 & 0.927 & 0.927 & 0.927 \\
$U_{11}(\mathrm{Ge}) / \AA^{2}$ & $0.016(2)$ & $0.029(3)$ & $0.0277(14)$ & $0.0338(18)$ & $0.038(2)$ \\
$U_{33}(\mathrm{Ge}) / \AA^{2}$ & $0.028(4)$ & $0.067(3)$ & $0.0256(19)$ & $0.039(11)$ & $0.039(10)$ \\
$U_{12}(\mathrm{Ge}) / \AA^{2}$ & $0.008(2)$ & $0.014(3)$ & $0.0139(14)$ & $0.0169(18)$ & $0.019(2)$ \\
$\mathrm{z}(\mathrm{Te}) / \AA$ & $0.76439(15)$ & $0.7608(3)$ & $0.7568(9)$ & $0.7566(13)$ & $0.752(2)$ \\
$U_{11}(\mathrm{Te}) / \AA^{2}$ & $0.015(3)$ & $0.016(3)$ & $0.0284(17)$ & $0.031(2)$ & $0.031(2)$ \\
$U_{33}(\mathrm{Te}) / \AA^{2}$ & $0.005(3)$ & $0.0098(16)$ & $0.062(7)$ & $0.046(18)$ & $0.044(17)$ \\
$U_{12}(\mathrm{Te}) / \AA^{2}$ & $0.007(3)$ & $0.008(3)$ & $0.0142(17)$ & $0.015(2)$ & $0.016(2)$ \\
$\mathrm{d}(\mathrm{Ge}-\mathrm{Te}) / \AA$ & $2.8273(8)$ & $2.8578(17)$ & $2.885(5)$ & $2.889(7)$ & $2.919(11)$ \\
$\mathrm{d}(\mathrm{Ge}-\mathrm{Te}) / \AA$ & $3.1667(10)$ & $3.148(2)$ & $3.123(6)$ & $3.123(9)$ & $3.094(13)$ \\
\hline
\end{tabular}

The displacement ellipsoids and full coordination at RT are shown in the representation of the crystal structure of Figure 3. Ge atoms present cigar-shaped displacement factors oriented along the c-axis direction.

The structural view at $298 \mathrm{~K}$ highlights the coordination polyhedron for Ge, which can be considered to be octahedral, although there are three short ( $2.83 \AA$ at RT) and three longer $(3.17 \AA$ at RT) distances (see Table 1), conferring a certain layered character to the crystal structure. The non-monotonous evolution of the displacement factors shown in Figure 3 (also in Table 1) is noteworthy. A large increment of the Ge ellipsoid is observed between 298 and $523 \mathrm{~K}$, which is then reduced for higher temperatures. In parallel, the Te ellipsoid also undergoes an irregular evolution, attaining a maximum size and anisotropy at $573 \mathrm{~K}$. The detailed analysis of the crystal structure also displays anomalies in this temperature region, always within the stability range of the rhombohedral phase, as shown in Figure 4. 


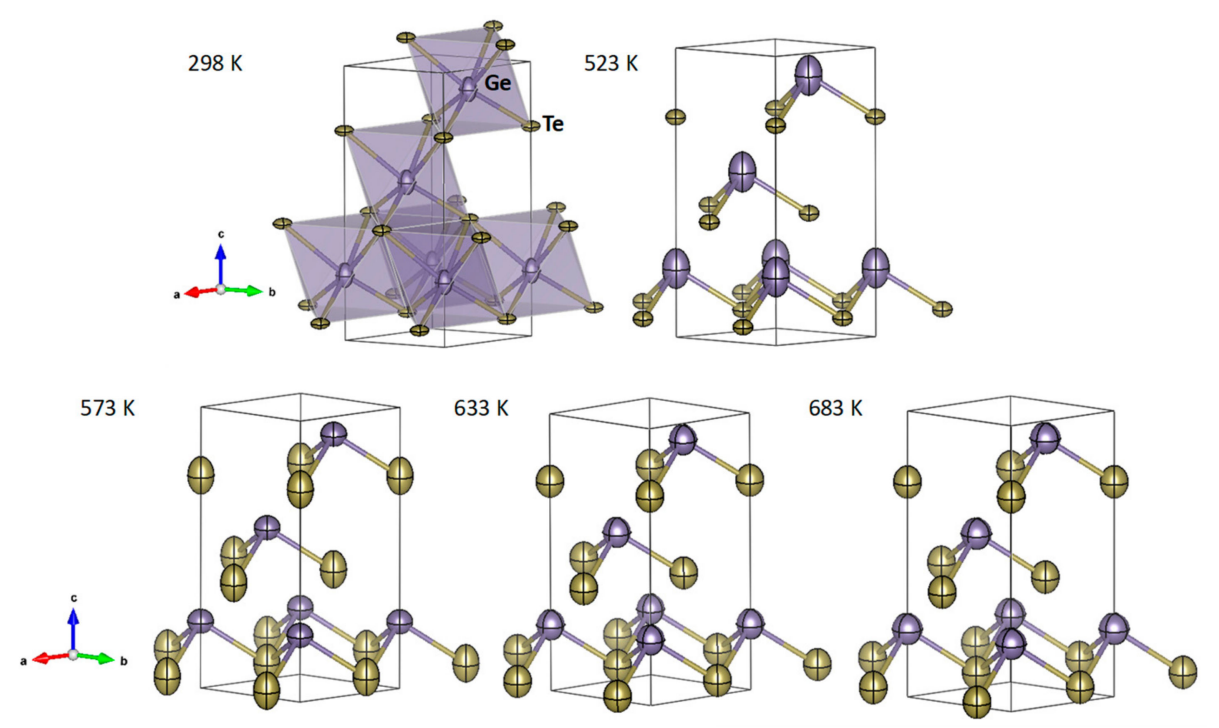

Figure 3. Views of the rhombohedral crystal structure, highlighting the evolution of the displacement parameters with temperature, from NPD data.
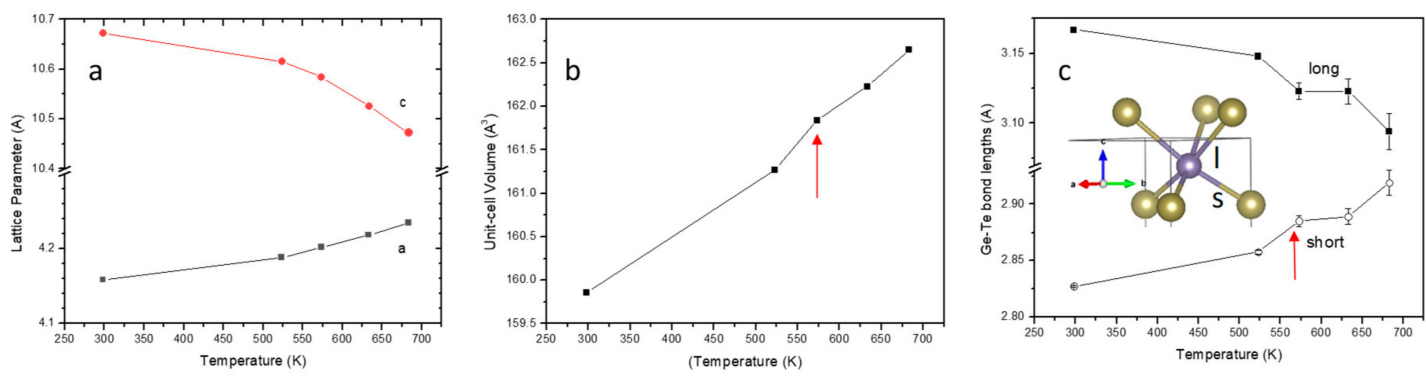

Figure 4. Thermal evolution of (a) a and c unit cell parameters in the hexagonal description of the rhombohedral structure, (b) unit-cell volume and (c) short and long Ge-Te distances of the GeTe 6 octahedra (see Figure 3 at 298 K), from NPD data. The arrow signals an anomaly in volume and bond-length expansion.

The unit-cell parameters ( $\mathrm{a}$ and $\mathrm{c}$ in the hexagonal description of the rhombohedral lattice) converge upon being heated as the structural transition is approached, but the unit-cell volume (Figure 4b) shows an anomaly (marked with an arrow) between 523 and $573 \mathrm{~K}$, which corresponds to an extra expansion of the short Ge-Te bonds, as shown in Figure 4c. This feature had not been reported before and it is concomitant with the abrupt modification of the displacement factors (Figure 3): those of Ge are decreasing, while Te experiences an enhanced displacement along the $\mathrm{c}$ axis of the ellipsoid, suggesting a looser chemical bond at this temperature corresponding to the longer bonding distances of around $2.89 \AA$ at $573 \mathrm{~K}$. Beyond this temperature, the thermal parameters behave as expected (Figure 3 and Table 1), progressively increasing with T. This anomaly, which is not correlated to any phase transition, has a repercussion in the physical properties, as we will see below.

For the high-temperature analysis, a SXRD experiment was performed at selected temperatures $(303,663,703,743$, and $783 \mathrm{~K})$. The RT pattern (303 K) confirms the already described rhombohedral structure, since the next studied temperature, $663 \mathrm{~K}$, already shows an incipient cubic structure. Presumably the measurement conditions, with the sample in a sealed quartz capillary, promote a slight shift of the transition temperature, which has been demonstrated to be very sensitive to chemical doping or external conditions [31-33]. Figure 5 shows a selected angular range that illustrates this phase transition. It is noteworthy that the extremely high resolution of the MSPD instrument involving the splitting of the characteristic (104) and (110) peaks at the $303 \mathrm{~K}$ rhombohedral phase. 


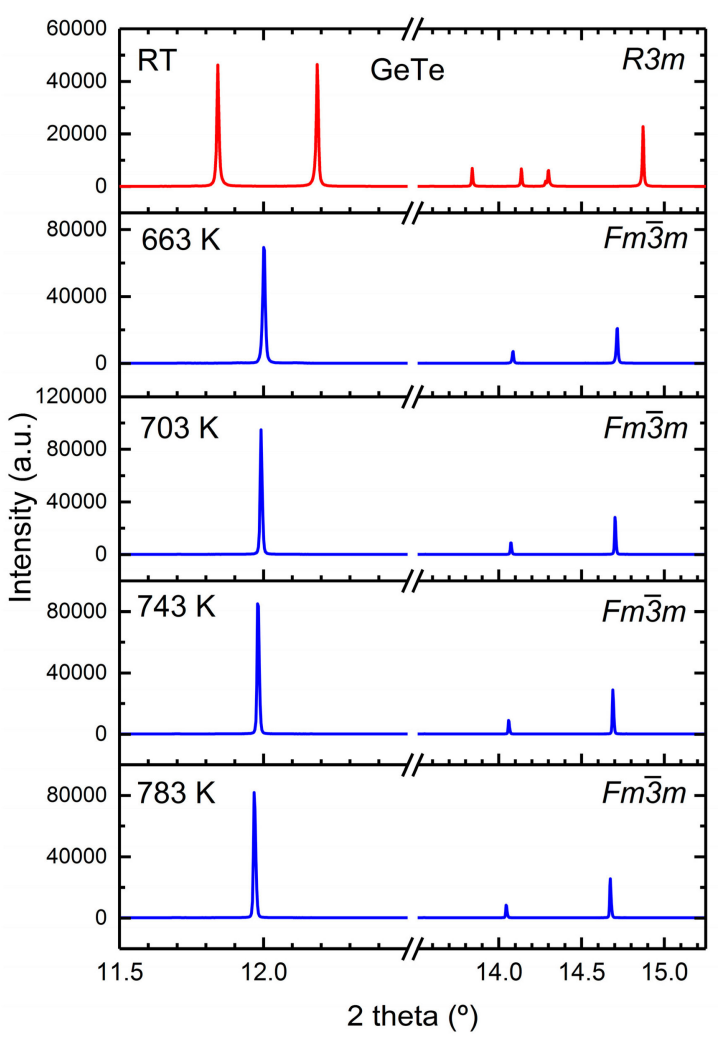

Figure 5. Thermal evolution of selected regions of the SXRD patterns of GeTe, in which a rhombohedral to cubic phase transition is evidenced.

At $663 \mathrm{~K}$ and above the cubic symmetry is defined in the space group $F m \overline{3} m$ (rock-salt structure): Ge atoms are located at $1 a(0,0,0)$ and Te atoms are placed at $1 c\left(\frac{1}{2}, \frac{1}{2}, \frac{1}{2}\right)$ Wyckoff site. Figure 6 plots the Rietveld refinements and selected temperatures, showing an excellent agreement between observed and calculated profiles, including an inset with a view of the cubic crystal structure above $663 \mathrm{~K}$. It also consists of a 3D framework of $\mathrm{GeTe}_{6}$ octahedra sharing edges along the three crystallographic directions, but these octahedra contain six equal Ge-Te distances. The main crystallographic data are listed in Table 2. Figure 7a plots the thermal variation of the cubic unit-cell parameter above the transition temperature, Figure $7 \mathrm{~b}$ depicts the evolution of the displacement factors for $\mathrm{Ge}$ and $\mathrm{Te}$, and Figure 7c shows the variation of the main interatomic distances both below (from NPD data) and above (from SXRD data) the phase transition.
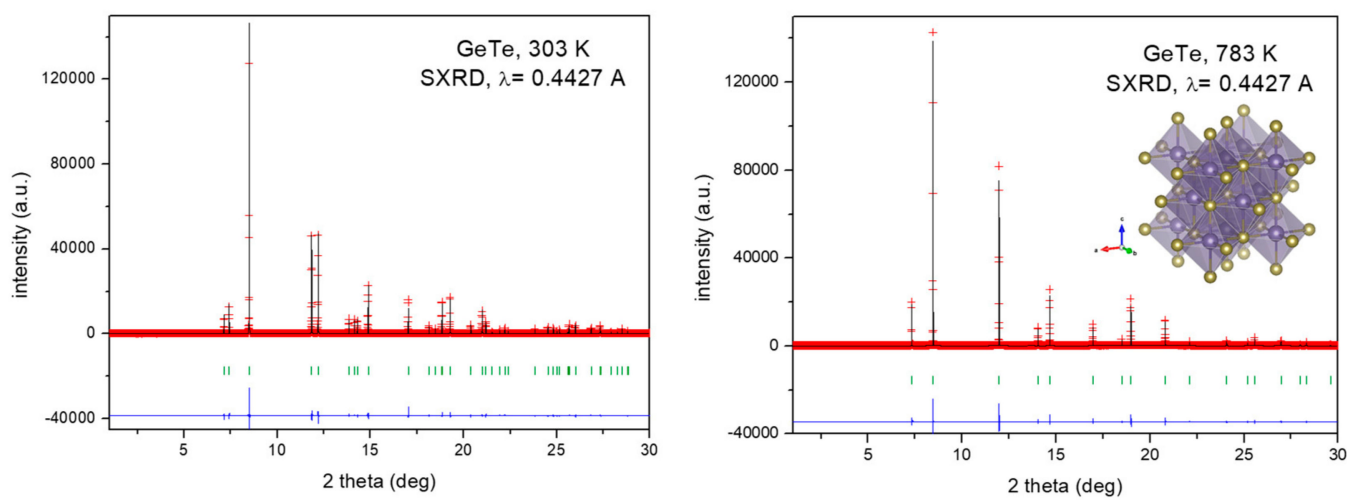

Figure 6. SXRD profiles after the Rietveld refinement of the crystal structure for the $303 \mathrm{~K}$ pattern (rhombohedral) and $783 \mathrm{~K}$ pattern (cubic). 
Table 2. Main structural parameters after the Rietveld refinement from NPD data for GeTe: rhombohedral $(R 3 m)$ at $303 \mathrm{~K}$ and cubic $(F m-3 m)$ at $663 \mathrm{~K}$ and above.

\begin{tabular}{cccccc}
\hline Temperature (K) & 303 & 663 & 703 & 743 & 783 \\
\hline$a / \AA$ & $4.16855(3)$ & $5.98590(6)$ & $5.99077(4)$ & $5.99615(5)$ & $6.00257(6)$ \\
$c / \AA$ & $10.66545(9)$ & & & & \\
$\mathrm{V} / \AA^{3}$ & $160.502(2)$ & $214.481(4)$ & $215.004(3)$ & $215.585(3)$ & $216.277(4)$ \\
$\mathrm{z}(\mathrm{Ge}) / \AA$ & 0.23760 & & & & \\
$\mathrm{z}(\mathrm{Te}) / \AA$ & $0.7642(6)$ & & & & \\
$U_{11}(\mathrm{Ge}) / \AA^{2}$ & $0.017(3)$ & $0.0509(19)$ & $0.0539(16)$ & $0.0562(18)$ & $0.0587(19)$ \\
$U_{33}(\mathrm{Ge}) / \AA^{2}$ & $0.015(9)$ & & & & \\
$U_{12}(\mathrm{Ge}) / \AA^{2}$ & $0.008(3)$ & & & & \\
$U_{11}(\mathrm{Te}) / \AA^{2}$ & $0.0106(14)$ & $0.0282(9)$ & $0.0309(8)$ & $0.0329(8)$ & $0.0348(9)$ \\
$U_{33}(\mathrm{Te}) / \AA^{2}$ & $0.015(4)$ & & & & \\
$U_{12}(\mathrm{Te}) / \AA^{2}$ & $0.0053(14)$ & & & & \\
$\mathrm{d}(\mathrm{Ge}-\mathrm{Te}) / \AA$ & $2.833(3)(\mathrm{x} 3)$ & $2.99295(3)(\mathrm{x} 6)$ & $2.99539(2)$ & $2.99808(2)$ & $3.00129(3)$ \\
$\mathrm{d}(\mathrm{Ge}-\mathrm{Te}) / \AA$ & $3.169(4)(\mathrm{x} 3)$ & & & & \\
\hline
\end{tabular}

Although in the cubic phase the displacement factors are isotropic by symmetry, it is remarkable that the Ge ones are consistently much larger than those of Te (Table 2, Figure 7b). This has been observed in other alloys crystallizing in rock-salt-type structures, like PbTe [34]. This effect can be attributed to the presence of the lone electron pair of $\mathrm{Ge}^{2+}$ ions (in an ionic model, assuming a total electron transfer from Ge to Te atoms). In a very symmetric unit cell, this electron lobe must be located at random in neighboring $\mathrm{GeTe}_{6}$ octahedra, leading to subtle shifts of the $\mathrm{Ge}$ ions and distinct $\mathrm{Ge}-\mathrm{Te}$ chemical bonds in an opposite location to that of the lone pairs, which indeed occupy a significant space in the crystal. In a diffraction domain, this effect of disorder or smearing of the scattering density is translated into an enhanced displacement factor for Ge atoms.

Furthermore, the occupancy and atomic mass weighted $U_{\text {iso }}$ displacement factors can typically be analyzed using a Debye-model with simple structures [35]. However, this analysis fails spectacularly as shown by the low quality of the least squares fit in Figure $7 \mathrm{~b}$ (yielding a nominal $220 \mathrm{~K}$ Debye temperature and rather high $0.13 \AA$ static disorder parameter). Interestingly, the $\mathrm{U}_{\text {iso }}$ of $\mathrm{Ge}$ on its own can be analyzed and well fit in terms of an Einstein-oscillator model. This model is often used to describe "rattlers" in thermoelectric materials as candidates for the so-called phonon glass electron crystal (PGEC) approach. We here raise the possibility that the Ge in Ge-deficient GeTe behaves somewhat akin to a rattler, with an Einstein energy of $8.3 \mathrm{meV}$ and a relatively modest $0.09 \AA$ Astatic disorder parameter. Similar analysis of the $\mathrm{U}_{\mathrm{iso}}$ of Te yields a negative squared static disorder parameter, indicating the limits of this approach.
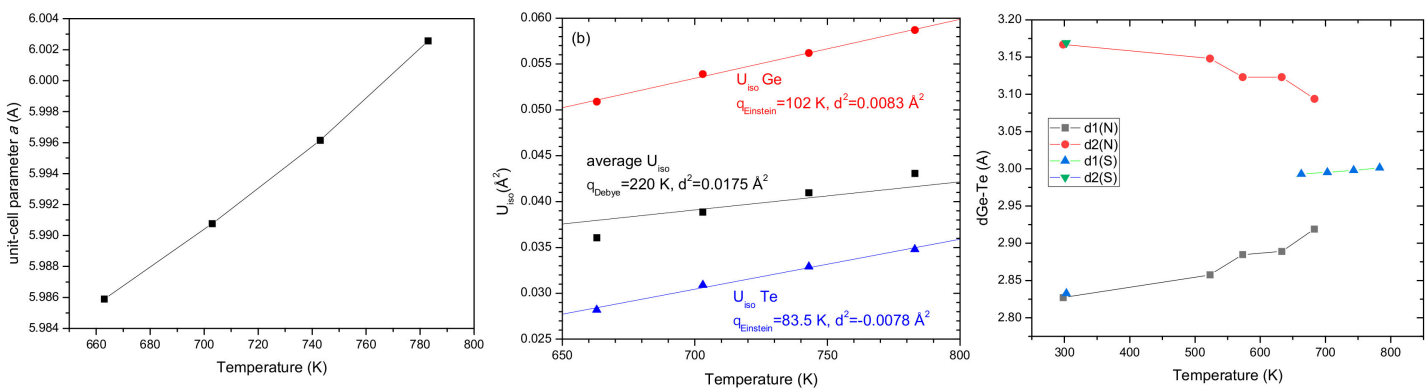

Figure 7. Thermal evolution of (a) unit cell parameters of the cubic phase at $663 \mathrm{~K}$ and above; (b) displacement factors, $\mathrm{U}_{\mathrm{iso}}$, for $\mathrm{Ge}$ (red circles) and Te (blue triangles) with corresponding Einstein oscillator analysis, and averaged $U_{\text {iso }}$ (black squares) with Debye analysis, indicating the best-fit Einstein and Debye temperatures and static disorder parameters; and (c) Ge-Te distances, including those obtained below the transition from NPD data, and above from SXRD data. 
The Seebeck coefficient measured in a home-made apparatus is shown in Figure 8. In a previous report [36] we showed that a high-carrier concentration of positive charge carriers in GeTe is due to Ge vacancies present in the crystal structure, as observed from NPD data. In agreement with this hypothesis, the temperature evolution is comparable to a heavily doped semiconductor and is dominated by hole mobility [37-39]. The maximum Seebeck coefficient $(120 \mu \mathrm{V} \mathrm{K}-1$ at $775 \mathrm{~K})$ is slightly reduced compared to other samples previously reported in the literature, with values closer to $150 \mu \mathrm{V} \mathrm{K}^{-1}$ at $700 \mathrm{~K}[13,14,40]$. The Hall-carrier concentration was reported as $1.72 \times 10^{21} \mathrm{~cm}^{-3}$ (at $250 \mathrm{~K}$ ), in the temperature range 200-350 K [33]. Note the conspicuous presence of a shoulder in the Seebeck curve, between 500 and $570 \mathrm{~K}$. This can be correlated to the structural feature observed from NPD data in the same temperature range (Figure 4c), consisting of a sudden increase of the 3 short distances of the $\mathrm{GeTe}_{6}$ octahedra, together with an increment of the Te displacement factors. In a qualitative way, the lengthening of the chemical bonds seems to suggest a reduced electron delocalization corresponding to the observed structural rearrangement. Most likely, this event in the structural evolution causes a sudden change in the electron-phonon scattering, which affects the Seebeck coefficient [41]. It is an open question whether this behavior is correlated to the significant Ge deficiency observed in our sample, since this anomaly is not reported in other works [5,42,43]. However, the aforementioned shoulder is present in other reports of the $S$ vs. T curve $[12,44,45]$ but it had never been correlated to structural features. We describe, therefore, a phenomenological relationship between crystal structure and properties that can be extrapolated to other GeTe-like materials.

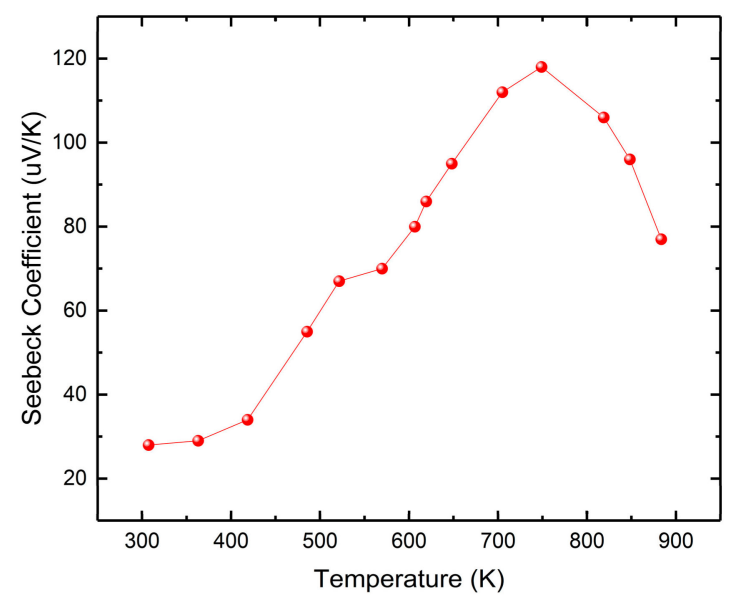

Figure 8. Seebeck coefficient thermal evolution, showing a shoulder between 500-570 K.

\section{Conclusions}

Polycrystalline GeTe ingots were prepared through arc-melting synthesis, yielding a pure yet Ge-deficient (7\% vacancies) material. The careful examination of the rhombohedral crystal structure evolution in the temperature range below the phase transition unveils an anomaly in the volume expansion, coupled with an abrupt stretching effect of the three short Ge-Te bonds that characterize the coordination polyhedra, as well as an increment of the Te displacement factors. This is concomitant with conspicuous changes in the Seebeck coefficient, presenting a shoulder in the temperature evolution curve. Above $699 \mathrm{~K}$ (according the DSC peak) or $663 \mathrm{~K}$ (from temperature-dependent SXRD data) the crystal structure is cubic, Fm-3m, showing a linear thermal expansion and significantly greater displacement factors for Ge vs. Te, attributed to the random distribution of the lone electron pair lobes in neighboring Ge positions.

Author Contributions: Conceptualization, J.A.A.; methodology, J.G., F.S.-S., N.M.N., M.T.F.-D., J.A.A.; software, J.G., M.T.F.-D., J.A.A.; validation M.T.F.-D., J.A.A.; formal analysis, M.T.F.-D., J.A.A.; investigation, J.G., F.S.-S, N.M.N., J.A.A.; resources, M.T.F.-D.; data curation, J.G., J.A.A.; writing—original draft preparation, F.S.-S., J.A.A.; writing-review and editing, J.G., F.S.-S., N.M.N., J.L.M., M.T.F.-D., J.A.A.; visualization, J.A.A.; supervision, 
N.M.N., J.A.A.; project administration, J.L.M., J.A.A.; funding acquisition, J.L.M., J.A.A. All authors have read and agreed to the published version of the manuscript.

Funding: This research was funded by Ministerio de Ciencia, Innovación y Universidades (MICIIN) for granting the projects MAT2017-84496-R and MAT2014-52405-C2-2-R.

Acknowledgments: We are grateful to the Spanish Ministry of Science, Innovation and Universities (MICIIN) for granting the projects MAT2017-84496-R and MAT2014-52405-C2-2-R, and ILL for making all facilities available for the neutron diffraction experiments. Javier Gainza thanks MICINN for granting the contract PRE2018-083398.

Conflicts of Interest: The authors declare no conflict of interest.

\section{References}

1. Okoye, C.M.I. Electronic and optical properties of SnTe and GeTe. J. Phys. Condens. Matter. 2002, 14, 8625. [CrossRef]

2. Bauer Pereira, P.; Sergueev, I.; Gorsse, S.; Dadda, J.; Müller, E.; Hermann, R.P. Lattice dynamics and structure of GeTe, SnTe and PbTe. Phys. Status Solidi 2013, 250, 1300-1307. [CrossRef]

3. Nukala, P.; Ren, M.; Agarwal, R.; Berger, J.; Liu, G.; Johnson, A.T.C.; Agarwal, R. Inverting polar domains via electrical pulsing in metallic germanium telluride. Nat. Commun. 2017, 8, 15033. [CrossRef] [PubMed]

4. Nukala, P.; Lin, C.-C.; Composto, R.; Agarwal, R. Ultralow-power switching via defect engineering in germanium telluride phase-change memory devices. Nat. Commun. 2016, 7, 10482. [CrossRef]

5. Li, J.; Zhang, X.; Chen, Z.; Lin, S.; Li, W.; Shen, J.; Witting, I.T.; Faghaninia, A.; Chen, Y.; Jain, A.; et al. Low-Symmetry Rhombohedral GeTe Thermoelectrics. Joule 2018, 2, 976-987. [CrossRef]

6. Goldsmid, H.J. Introduction to Thermoelectricity; Springer: Berlin, Germany, 2017; ISBN 9783540687573.

7. Nolas, G.S.; Sharp, J.; Goldsmid, H.J. Thermoelectrics: Basic Principles and New Materials Developments; Springer: New York, NY, USA, 2001; ISBN 9783642074516.

8. Bell, L.E. Cooling, heating, generating power, and recovering waste heat with thermoelectric systems. Science 2008, 321, 1457-1461. [CrossRef]

9. Snyder, G.J.; Toberer, E.S. Complex thermoelectric materials. Nat. Mater. 2008, 7, 105-114. [CrossRef]

10. Kolomoets, N.V.; Lev, E.Y.; Sysoeva, L.M. Electrical properties and valence band structure of germanium telluride. Sov. Phys. Solid State 1964, 6, 551-556.

11. Akola, J.; Jones, R.O. Binary alloys of Ge and Te: Order, voids, and the eutectic composition. Phys. Rev. Lett. 2008, 100, 21-24. [CrossRef]

12. Perumal, S.; Samanta, M.; Ghosh, T.; Shenoy, U.S.; Bohra, A.K.; Bhattacharya, S.; Singh, A.; Waghmare, U.V.; Biswas, K. Realization of High Thermoelectric Figure of Merit in GeTe by Complementary Co-doping of Bi and In. Joule 2019, 3, 2565-2580. [CrossRef]

13. Gelbstein, Y.; Dado, B.; Ben-Yehuda, O.; Sadia, Y.; Dashevsky, Z.; Dariel, M.P. Highly efficient Ge-Rich GexPb1-xTe thermoelectric alloys. J. Electron. Mater. 2010, 39, 2049-2052. [CrossRef]

14. Gelbstein, Y.; Ben-Yehuda, O.; Pinhas, E.; Edrei, T.; Sadia, Y.; Dashevsky, Z.; Dariel, M.P. Thermoelectric Properties of (Pb,Sn,Ge)Te-Based Alloys. J. Electron. Mater. 2009, 38, 1478-1482. [CrossRef]

15. Lee, H.S.; Kim, B.-S.; Cho, C.-W.; Oh, M.-W.; Min, B.-K.; Park, S.-D.; Lee, H.-W. Herringbone structure in GeTe-based thermoelectric materials. Acta Mater. 2015, 91, 83-90. [CrossRef]

16. Vermeulen, P.A.; Kumar, A.; ten Brink, G.H.; Blake, G.R.; Kooi, B.J. Unravelling the Domain Structures in GeTe and LaAlO 3. Cryst. Growth Des. 2016, 16, 5915-5922. [CrossRef]

17. Matsunaga, T.; Fons, P.; Kolobov, A.V.; Tominaga, J.; Yamada, N. The order-disorder transition in GeTe: Views from different length-scales. Appl. Phys. Lett. 2011, 99, 231907. [CrossRef]

18. Chattopadhyay, T.; Boucherle, J.X.; VonSchnering, H.G. Neutron diffraction study on the structural phase transition in GeTe. J. Phys. C Solid State Phys. 1987, 20, 1431-1440. [CrossRef]

19. Wdowik, U.D.; Parlinski, K.; Rols, S.; Chatterji, T. Soft-phonon mediated structural phase transition in GeTe. Phys. Rev. B 2014, 89, 1-7. [CrossRef]

20. Fons, P.; Kolobov, A.V.; Krbal, M.; Tominaga, J.; Andrikopoulos, K.S.; Yannopoulos, S.N.; Voyiatzis, G.A.; Uruga, T. Phase transition in crystalline GeTe: Pitfalls of averaging effects. Phys. Rev. B 2010, 82, 155209. [CrossRef] 
21. Tran, X.Q.; Hong, M.; Maeno, H.; Kawami, Y.; Toriyama, T.; Jack, K.; Chen, Z.-G.; Zou, J.; Matsumura, S.; Dargusch, M.S. Real-time observation of the thermally-induced phase transformation in GeTe and its thermal expansion properties. Acta Mater. 2019, 165, 327-335. [CrossRef]

22. Xu, M.; Lei, Z.; Yuan, J.; Xue, K.; Guo, Y.; Wang, S.; Miao, X.; Mazzarello, R. Structural disorder in the high-temperature cubic phase of GeTe. RSC Adv. 2018, 8, 17435-17442. [CrossRef]

23. Sist, M.; Kasai, H.; Hedegaard, E.M.J.; Iversen, B.B. Role of vacancies in the high-temperature pseudodisplacive phase transition in GeTe. Phys. Rev. B 2018, 97, 94116. [CrossRef]

24. Shportko, K.; Kremers, S.; Woda, M.; Lencer, D.; Robertson, J.; Wuttig, M. Resonant bonding in crystalline phase-change materials. Nat. Mater. 2008, 7, 653. [CrossRef] [PubMed]

25. Boschker, J.E.; Wang, R.; Calarco, R. GeTe: A simple compound blessed with a plethora of properties. CrystEngComm 2017, 19, 5324-5335. [CrossRef]

26. Fauth, F.; Boer, R.; Gil-Ortiz, F.; Popescu, C.; Vallcorba, O.; Peral, I.; Fullà, D.; Benach, J.; Juanhuix, J. The crystallography stations at the Alba synchrotron. Eur. Phys. J. Plus 2015, 130, 160. [CrossRef]

27. Rietveld, H.M. A profile refinement method for nuclear and magnetic structures. J. Appl. Crystallogr. 1969, 2, 65-71. [CrossRef]

28. Rodríguez-Carvajal, J. Recent advances in magnetic structure determination by neutron powder diffraction. Phys. B Phys. Condens. Matter. 1993, 192, 55-69. [CrossRef]

29. Iwanaga, S.; Toberer, E.S.; LaLonde, A.; Snyder, G.J. A high temperature apparatus for measurement of the Seebeck coefficient. Rev. Sci. Instrum. 2011, 82, 063905. [CrossRef]

30. Perumal, S.; Roychowdhury, S.; Negi, D.S.; Datta, R.; Biswas, K. High Thermoelectric Performance and Enhanced Mechanical Stability of p-type Ge1-xSbxTe. Chem. Mater. 2015, 27, 7171-7178. [CrossRef]

31. Gainza, J.; Serrano-Sánchez, F.; Nemes, N.M.; Dura, O.J.; Martínez, J.L.; Alonso, J.A. Lower temperature of the structural transition, and thermoelectric properties in Sn-substituted GeTe. Mater. Today. Proc. 2019. submitted for publication.

32. Wu, D.; Zhao, L.D.; Hao, S.; Jiang, Q.; Zheng, F.; Doak, J.W.; Wu, H.; Chi, H.; Gelbstein, Y.; Uher, C.; et al. Origin of the high performance in GeTe-based thermoelectric materials upon Bi2Te3 doping. J. Am. Chem. Soc. 2014, 136, 11412-11419. [CrossRef]

33. Chatterji, T.; Kumar, C.M.N.; Wdowik, U.D. Anomalous temperature-induced volume contraction in GeTe. Phys. Rev. B 2015, 91, 054110. [CrossRef]

34. Gainza, J.; Serrano-Sánchez, F.; Biskup, N.; Nemes, N.M.; Martínez, J.L.; Fernández-Díaz, M.T.; Alonso, J.A. Influence of Nanostructuration on PbTe Alloys Synthesized by Arc-Melting. Materials 2019, 12, 3783. [CrossRef] [PubMed]

35. Mi, J.-L.; Christensen, M.; Nishibori, E.; Iversen, B.B. Multitemperature crystal structures and physical properties of the partially filled thermoelectric skutterudites M0.1Co4Sb12 (M = La,Ce,Nd,Sm, Yb and Eu). Phys. Rev. B 2011, 84, 064114. [CrossRef]

36. Serrano-Sánchez, F.; Funes, M.; Nemes, N.M.; Dura, O.J.; Martínez, J.L.; Prado-Gonjal, J.; Fernández-Díaz, M.T.; Alonso, J.A. Low lattice thermal conductivity in arc-melted GeTe with Ge-deficient crystal structure. Appl. Phys. Lett. 2018, 113, 1-5. [CrossRef]

37. Gascoin, F.; Ottensmann, S.; Stark, D.; Haïle, S.M.; Snyder, G.J. Zintl phases as thermoelectric materials: Tuned transport properties of the compounds CaxYb1-xZn2Sb2. Adv. Funct. Mater. 2005, 15, 1860-1864. [CrossRef]

38. Peng, K.; Lu, X.; Zhan, H.; Hui, S.; Tang, X.; Wang, G.; Dai, J.; Uher, C.; Wang, G.; Zhou, X. Broad temperature plateau for high ZTs in heavily doped p-type SnSe single crystals. Energy Environ. Sci. 2016, 9, 454-460. [CrossRef]

39. Li, Y.; Qiu, P.; Xiong, Z.; Chen, J.; Nunna, R.; Shi, X.; Chen, L. Electrical and thermal transport properties of YbxCo4Sb12 filled skutterudites with ultrahigh carrier concentrations. AIP Adv. 2015, 5, 117239. [CrossRef]

40. Levin, E.M.; Besser, M.F.; Hanus, R. Electronic and thermal transport in GeTe: A versatile base for thermoelectric materials. J. Appl. Phys. 2013, 114, 083713. [CrossRef]

41. May, A.F.; Toberer, E.S.; Saramat, A.; Snyder, G.J. Characterization and analysis of thermoelectric transport in n -type Ba8 Ga16-x Ge30+x. Phys. Rev. B 2009, 80, 1-12. [CrossRef]

42. Lee, J.K.; Oh, M.W.; Kim, B.S.; Min, B.K.; Lee, H.W.; Park, S.D. Influence of Mn on crystal structure and thermoelectric properties of GeTe compounds. Electron. Mater. Lett. 2014, 10, 813-817. [CrossRef] 
43. Dong, J.; Sun, F.; Tang, H.; Pei, J.; Zhuang, H.; Hu, H.; Zhang, B.; Pan, Y.; Li, J. Medium-temperature thermoelectric GeTe: Vacancy suppression and band structure engineering leading to high performance. Energy Environ. Sci. 2019, 12, 1396-1403. [CrossRef]

44. Perumal, S.; Roychowdhury, S.; Biswas, K. High performance thermoelectric materials and devices based on GeTe. J. Mater. Chem. C 2016, 4, 7520-7536. [CrossRef]

45. Li, J.; Zhang, X.; Lin, S.; Chen, Z.; Pei, Y. Realizing the High Thermoelectric Performance of GeTe by Sb-Doping and Se-Alloying. Chem. Mater. 2017, 29, 605-611. [CrossRef]

(C) 2019 by the authors. Licensee MDPI, Basel, Switzerland. This article is an open access article distributed under the terms and conditions of the Creative Commons Attribution (CC BY) license (http://creativecommons.org/licenses/by/4.0/). 